\title{
EXISTENCE OF INTEGRAL MANIFOLDS FOR IMPULSIVE DIFFERENTIAL EQUATION WITH SMALL PARAMETER
}

\author{
S. KOSTADINOV ${ }^{1}$, K. SCHNEIDER ${ }^{2}$, M. VENKOVA ${ }^{3}$
}

\begin{abstract}
Applying the Banach fixed-point theorem, sufficient conditions for the existence of bounded manifolds of Lipschitz type are found.
\end{abstract}

\section{Introduction}

Many papers and monographs devoted to impulsive differential equations have appeared in the last 10 years (for example [1], [3], [4] and [5], where further references can be found). On the other hand, the theory of the differential equations with small parameter makes rapid development because it can be successfully applied in physics, mechanice etc. By generalizing of some results from [6] the present paper is trying to connect these two impoartant fields of study (see also [2]).

\section{Statement of the problem}

Consider the impulsive differential equation:

$$
\begin{aligned}
\frac{d x}{d t} & =F(t, x, y, \varepsilon) \quad\left(t \geq 0, t \neq t_{n}\right) \\
\Delta x / t \neq t_{n} & =Q_{n}\left(x\left(t_{n}\right), y\left(t_{n}\right), \varepsilon\right) \quad(n \in N) \\
\varepsilon \frac{d y}{d t} & =A(t, x) y+G(t, x, y, \varepsilon) \quad\left(t \geq 0, t \neq t_{n}\right) \\
\varepsilon \Delta y / t \neq t_{n} & =P_{n}(x) y\left(t_{n}\right)+R_{n}\left(x\left(t_{n}\right), y\left(t_{n}\right), \varepsilon\right) \quad(n \in N)
\end{aligned}
$$

where $x \in R^{k}, y \in R^{m}, \varepsilon \geq 0$ and $A(t, x), P_{n}$ are $(m, m)$-matrices $(t \geq 0, n \in N)$.

We introduce the following conditions:

H1. There exist numbers $l>0$ and $m \in N$ such that each interval on $R^{+}$with length $l$ contains not more than $m$ points of the sequence $\left\{t_{n}\right\}_{n=0}^{+\infty}, t_{i+1}>t_{i}$ and $\inf _{i \in N}\left(t_{i+1}-t_{i}\right)=$ $p>0$.

Received February 20, 1998.

1991 Mathematics Subject Classification. 34A37, 47H10.

Key words and phrases. Impulsive differential equations, bounded integral manifolds of Lipschitz type. 
H2. There exist $\varepsilon_{0} \geq 0$ and $C \geq 0$ such that

$$
\begin{aligned}
& \left\|F\left(t, x_{1}, y_{1}, \varepsilon\right)-F\left(t, x_{2}, y_{2}, \varepsilon\right)\right\| \leq C\left(\left\|x_{1}-x_{2}\right\|+\left\|y_{1}-y_{2}\right\|\right), \\
& \left\|Q_{n}\left(x_{1}, y_{1}, \varepsilon\right)-Q_{n}\left(x_{2}, y_{2}, \varepsilon\right)\right\| \leq C\left(\left\|x_{1}-x_{2}\right\|+\left\|y_{1}-y_{2}\right\|\right), \\
& \left\|Q_{n}(x, y, \varepsilon)\right\| \leq C_{p} \\
& \mid A\left(t, x_{1}\right)-A\left(t, x_{2}\right) \| \leq C\left(\left|t_{1}-t_{2}\right|+\left\|x_{1}-x_{2}\right\|\right), \\
& \left\|G\left(t, x_{1}, y_{1}, \varepsilon\right)-G\left(t, x_{2}, y_{2}, \varepsilon\right)\right\| \leq C\left(\left\|x_{1}-x_{2}\right\|+\left\|y_{1}-y_{2}\right\|\right)\left(\left(\max \left\{\left\|y_{1}\right\|,\left\|y_{2}\right\|\right\}\right)^{2}+\varepsilon^{2}\right), \\
& \|G(t, x, y, \varepsilon)\| \leq C\left(\|y\|^{2}+\varepsilon\right), \\
& \left\|R_{n}\left(x_{1}, y_{1}, \varepsilon\right)-R_{n}\left(x_{2}, y_{2}, \varepsilon\right)\right\| \leq C\left(\left(\max \left\{\left\|y_{1}\right\|,\left\|y_{2}\right\|\right\}\right)^{2}+\varepsilon^{2}\right)\left(\left\|x_{1}-x_{2}\right\|+\left\|y_{1}-y_{2}\right\|\right), \\
& \left\|R_{n}(x, y, \varepsilon)\right\| \leq C\left(\|y\|^{4}+\varepsilon^{4}\right), \\
& \left\|P_{n}\left(x_{1}\right)-P_{n}\left(x_{2}\right)\right\| \leq C\left\|x_{1}-x_{2}\right\|
\end{aligned}
$$

for any $x, x_{1}, x_{2} \in R^{k}, y, y_{1}, y_{2} \in R^{m}, t \geq 0, \varepsilon \in\left[0, \varepsilon_{0}\right]$ and $n \in N$.

H3. The eigenvalues of $A(t, x)$ satisfy the inequation

$$
\operatorname{Re} \lambda_{i}(t, x) \leq-2 \gamma \quad i=1, \ldots, m
$$

for some $\gamma>0$ and $\prod_{i=1}^{\infty}\left\|I+P_{i}\right\|<\infty$.

Under the assumptions $\mathrm{H} 1, \mathrm{H} 2$ and $\mathrm{H} 3$ we are going to prove the existence of integral manifold for equation $\left(1,1^{\prime}\right)$.

\section{Main results}

Theorem 1. Let the conditions H1, H2, and H3 hold. Then exist $\varepsilon^{*} \in\left(0, \varepsilon_{0}\right]$ and positive constants $D$ and $\Delta$ such that for every $\varepsilon \in\left(0, \varepsilon^{*}\right]$ the equation $\left(1,1^{\prime}\right)$ has an unique integral manifold $y=H^{*}(t, x, \varepsilon)$ with the following properties:

1. $\left\|H^{*}(t, x, \varepsilon)\right\| \leq D$.

2. $\left\|H^{*}\left(t, x_{1}, \varepsilon\right)-H^{*}\left(t, x_{2}, \varepsilon\right)\right\| \leq \Delta\left\|x_{1}-x_{2}\right\|$.

3. $\frac{d x}{d t}=F\left(t, x, H^{*}(t, x, \varepsilon), \varepsilon\right) \quad\left(t \geq 0, t \neq t_{n}\right)$

$\Delta x / t=t_{n}=Q_{n}\left(x\left(t_{n}\right), H^{*}\left(t_{n}, x\left(t_{n}\right), \varepsilon\right), \varepsilon\right) \quad(n \in N)$.

In advance we will prove the following lemma:

Lemma 1. Let $A(t)$ be bounded $m \times m$ matrix for every $t \geq 0$ and Lipschitz with constant $q$. Besides let the eigenvalues of $A(t)$ satisfy the inequation

$$
\operatorname{Re}_{i}(t) \leq-2 \gamma \quad i=1, \ldots, m
$$

for some $\gamma>0$ and $\prod_{i=1}^{\infty}\left\|I+P_{i}\right\|<\infty$. Then exist positive constants $L$ and $\varepsilon_{1}$ such that the Cauchy operator $W(\tau, t, \varepsilon)$ of the equation

$$
\begin{aligned}
\varepsilon \frac{d z}{d t} & =A(t) z & & \left(t \geq 0, t \neq t_{n}\right) \\
\varepsilon \Delta z / t=t_{n} & =P_{n}(z) & & (n \in N)
\end{aligned}
$$


satisfies the inequation

$$
\|W(\tau, t, \varepsilon)\| \leq K e^{-\frac{\gamma}{\varepsilon}(\tau-t)}
$$

for any $0 \leq t \leq \tau<\infty, \varepsilon \in\left[0, \varepsilon_{1}\right]$ and a constant $K>0$.

Proof. At first let prove (see [6]) that the Cauchy operator $U(\tau, t, \varepsilon)$ of the equation

$$
\varepsilon \frac{d z}{d t}=A(t) z
$$

satisfies the estimate

$$
\|U(\tau, t, \varepsilon)\| \leq L e^{-\frac{\gamma}{\varepsilon}(\tau-t)} e^{-\left[\frac{\gamma}{2 \varepsilon}-\sqrt{\frac{K q}{\varepsilon}}\right](\tau-t)}
$$

for some $L \geq 0$ and $\varepsilon \in\left[0, \varepsilon_{1}^{\prime}\right]$. For any fixed $u \in R$ the following equation holds true:

$$
U(\tau, t, \varepsilon)=e^{\frac{1}{\varepsilon} A(u)(\tau-t)}+\frac{1}{\varepsilon} \int_{t}^{\tau} e^{\frac{1}{\varepsilon} A(u)(\tau-s)}[A(s)-A(u)] U(s, t, \varepsilon) d s
$$

Since $\|A(t)\|$ is bounded and $\operatorname{Re} \lambda_{i}(t) \leq-2 \gamma$ for some $L \geq 0$, we have

$$
\left\|e^{\frac{1}{\varepsilon} A(u)(\tau-t)}\right\| \leq L e^{-\frac{3 \gamma}{3 \varepsilon}(\tau-t)}
$$

for all $0 \leq t \leq \tau<\infty, \varepsilon \in\left[0, \varepsilon_{0}\right]$ and $u \in R$. Now let $u=\tau$, then

$$
\|U(\tau, t, \varepsilon)\|=L e^{-\frac{3 \gamma}{2 \varepsilon}(\tau-t)}+\frac{L q}{\varepsilon} \int_{t}^{\tau} e^{\frac{3 \gamma}{2 \varepsilon}(s-t)}\|U(s, t, \varepsilon)\|(\tau-s) d s .
$$

Hence

$$
\|U(\tau, t, \varepsilon)\| e^{\frac{3 \gamma}{2 \varepsilon}(\tau-t)}=L+\frac{L q}{\varepsilon} \int_{t}^{\tau} e^{\frac{3 \gamma}{2 \varepsilon}(s-t)}\|U(s, t, \varepsilon)\|(\tau-s) d s .
$$

Let $\nu(s)=e^{\frac{3 \gamma}{2 \varepsilon}(s-\hat{t})}\|U(s, t, \varepsilon)\|$. It is not hard to check that

$$
\nu(\tau) \leq \nu_{0}(\tau)=\frac{L}{2}\left[e^{\sqrt{\frac{L q}{\varepsilon}}(\tau-t)}+e^{\left.-\sqrt{\frac{L q}{\varepsilon}}\right)(\tau-t)}\right] .
$$

This means that

$$
\|U(\tau, t, \varepsilon)\| \leq \frac{L}{2} e^{-\frac{\gamma}{\varepsilon}(\tau-t)}\left[e^{-\left(\frac{\gamma}{2 \varepsilon}-\sqrt{\frac{L q}{\varepsilon}}\right)(\tau-t)}+e^{-\left(\frac{\gamma}{2 \varepsilon}+\sqrt{\frac{L q}{\varepsilon}}\right)(\tau-t)}\right] .
$$

Consequently for $\varepsilon_{1}^{\prime}=\frac{\gamma^{2}}{4 L q}$ and $\varepsilon \in\left[0, \varepsilon_{1}^{\prime}\right]$ (3) holds true.

Consider now the impulsive differential equation (2). In [3] is shown that

$$
\begin{gathered}
W(\tau, t, \varepsilon)=U\left(\tau, t_{j+k}, \varepsilon\right)\left(I+P_{j+k}\right) U\left(t_{j+k}, t_{j+k-1}, \varepsilon\right)\left(I+P_{j+k-1}\right) \\
\times \cdots \times\left(I+P_{j}\right) U\left(t_{j}, t, \varepsilon\right) \quad\left(0 \leq t \leq t_{j}<\cdots<t_{j+k} \leq \tau<\infty\right) .
\end{gathered}
$$

Set

$$
\mu=\frac{\gamma}{2 \varepsilon}-\sqrt{\frac{L q}{\varepsilon}} .
$$


Hence

$$
\begin{gathered}
\|W(\tau, t, \varepsilon)\| \leq L e^{-\frac{\gamma}{\varepsilon}(\tau-t)} e^{-\mu(\tau-t)}\left(\prod_{i=0}^{k} L\left\|I+P_{j+i}\right\|\right) \\
\leq L^{3} e^{-\frac{\gamma}{\varepsilon}(\tau-t)} e^{\mu(\tau-t)+(k-1) \ln L+\sum_{i=0}^{k} \ln \left\|I+P_{i+j}\right\|}
\end{gathered}
$$

There exists $\varepsilon_{1}$ such that for $\varepsilon \in\left[0, \varepsilon_{1}\right]$ the estimate

$$
\ln L \leq\left(\frac{\gamma}{2 \varepsilon}-\sqrt{\frac{L q}{\varepsilon}}\right) p
$$

holds true. Hence

$$
(k-1) \ln L<(k-1) \mu p<\mu(\tau-t)
$$

and consequently

$$
\|W(\tau, t, \varepsilon)\| \leq L e^{-\frac{\gamma}{\varepsilon}(\tau-t)} \prod_{i=1}^{k}\left\|I+P_{j+i}\right\| \leq L e^{-\frac{\gamma}{\varepsilon}(\tau-t)} \prod_{i=1}^{\infty}\left\|I+P_{i}\right\| \leq K e^{-\frac{\gamma}{\varepsilon}(\tau-t)} .
$$

Let $M(\Delta, D)$ be the set of all functions $f: \Omega \rightarrow R^{m}$, where $\Omega=\left\{t \geq 0, x \in R^{k}, \varepsilon \in\right.$ $\left.\left[0, \varepsilon_{0}\right]\right\}, f$ are continuous for $t \neq t_{i}$, left-hand continuous in $t_{i}(i \in N)$, and satisfy the following conditions

$$
\begin{aligned}
& \left\|f(t, x, \varepsilon)-f\left(t, x^{\prime}, \varepsilon\right)\right\| \leq \Delta\left\|x-x^{\prime}\right\|, \\
& \|f(t, x, \varepsilon)\| \leq D
\end{aligned}
$$

Consider the function

$$
\rho\left(f, f^{*}\right)=\sup _{(t, x, \varepsilon) \in \Omega}\left\|f(t, x, \varepsilon)-f^{*}(t, x, \varepsilon)\right\|=\left\|\left|f-f^{*}\right|\right\|
$$

It is not hard to see that $(M(\Delta, D), \rho)$ is a complete metric space.

Proof of Theorem 1. Let $H \in M(\Delta, d)$. Then using $\mathrm{H} 2$ we obtain

$$
\begin{aligned}
& \left\|F(t, x, H(t, x, \varepsilon), \varepsilon)-F\left(t, x^{*}, H\left(t, x^{*}, \varepsilon\right), \varepsilon\right)\right\| \\
\leq & C\left(\left\|x-x^{*}\right\|+\left\|H(t, x, \varepsilon)-H\left(t, x^{*}, \varepsilon\right)\right\|\right) \leq C(1+\Delta)\left\|x-x^{*}\right\|\left(x, x^{*} \in R^{k}, t \geq 0\right)
\end{aligned}
$$

Similarly

$$
\left\|Q_{n}(x, H(t, x, \varepsilon), \varepsilon)-Q_{n}\left(x^{*}, H\left(t, x^{*}, \varepsilon\right), \varepsilon\right)\right\| \leq C(1+\Delta)\left\|x-x^{*}\right\| \quad\left(x, x^{*} \in R^{n}, n \in N\right)
$$

The equation

$$
\begin{aligned}
\frac{d x}{d t} & =F(t, x, H(t, x, \varepsilon), \varepsilon) \quad\left(t \geq 0, t \neq t_{n}\right) \\
\Delta x / t=t_{n} & =Q_{n}\left(x\left(t_{n}\right), H\left(t_{n}, x\left(t_{n}\right), \varepsilon\right), \varepsilon\right) \quad(n \in N) \\
x(0) & =\xi
\end{aligned}
$$


has an unique solution $\varphi(t)$ for any $\xi \in R^{m}$. Let fix a number $\tau \geq 0$ and denote $\varphi(\tau)=x$. Since later on we use the abbreviation $\phi(t)=\Phi(t, \tau, x, \varepsilon / H)$. Notice that the function $\varphi(t)$ is a solution of the equation

$\varphi(t)=x-\int_{t}^{\tau} F(s, \varphi(s), H(s, \varphi(s), \varepsilon), \varepsilon) d s-\sum_{t<t_{i}<\tau} Q_{i}\left(\varphi\left(t_{i}\right), H\left(t_{i}, \varphi\left(t_{i}\right), \varepsilon, \varepsilon\right) \quad(0 \leq t \leq \tau)\right.$

If $\varphi(t)$ and $\varphi^{*}(t)$ are two solutions of $(4)-\left(4^{\prime \prime}\right)$ and $\phi(\tau)=x, \varphi^{*}(\tau)=x^{*}$, then the following inequality is valid

$$
\begin{aligned}
& \quad\left\|\varphi(t)-\varphi^{*}(t)\right\| \leq\left\|x-x^{*}\right\| \\
& +\int_{t}^{\tau}\left\|F(s, \varphi(s), H(s, \varphi(s), \varepsilon), \varepsilon)-F\left(s, \varphi^{*}(s), H^{*}\left(s, \varphi^{*}(s), \varepsilon\right), \varepsilon\right)\right\| d s \\
& +\sum_{i<t_{i}<\tau}\left\|Q_{i}\left(\varphi\left(t_{i}\right), H\left(t, \varphi\left(t_{i}\right), \varepsilon\right), \varepsilon\right)-Q_{n}\left(\operatorname{varphi}^{*}\left(t_{i}\right), H^{*}\left(t_{i}, \varphi^{*}\left(t_{i}\right), \varepsilon\right), \varepsilon\right)\right\| \\
& \leq\left\|x-x^{*}\right\|+C \int_{t}^{\tau}\left((1+\Delta)\left\|\varphi(s)-\varphi^{*}(s)\right\|+\left\|\left|H-H^{*}\right|\right\|\right) d s \\
& +C \sum_{t<t_{i}<\tau}\left((1+\Delta)\left\|\varphi\left(t_{i}\right)-\varphi^{*}\left(t_{i}\right)\right\|+\left\|\mid H-H^{*}\right\|\right) \quad(0 \leq t \leq \tau)
\end{aligned}
$$

Applying the integral inequality from [3], we obtain the estimate

$$
\left\|\varphi(t)-\varphi^{*}(t)\right\| \leq\left(\left\|x-x^{*}\right\|+\frac{\left\|\left|H-H^{*}\right|\right\|}{1+\Delta}\right)(1+a)^{k(t, \tau)}-\frac{\left\|\left|H-H^{*}\right|\right\|}{1+\Delta}
$$

where $k(t, \tau)$ is the number of the impulse points in $[t, \tau)$, and $a$ is an arbitrary constant fulfilling $a \geq C(1+\Delta)$.

Consider the equation

$$
\begin{aligned}
\varepsilon \frac{d y}{d t} & =A(t, \varphi(t)) y+G(t, \varphi(t), y, \varepsilon) \quad\left(t \geq 0, t \neq t_{n}\right) \\
\varepsilon \Delta y /_{t=t_{n}} & =P_{n}\left(\varphi\left(t_{n}\right)\right) y+R_{n}\left(\varphi\left(t_{n}\right), y\left(t_{n}\right)\right) \quad(n \in N)
\end{aligned}
$$

At first we are going to prove that the Cauchy matrix $U=U_{\varphi}(\tau, t, \varepsilon)$ of the equation

$$
\begin{aligned}
\varepsilon \frac{d y}{d t} & =A(t, \varphi(t)) y & & \left(t \geq 0, t \neq t_{n}\right) \\
\left.\varepsilon \Delta y\right|_{t=t_{n}} & =P_{n}\left(\varphi\left(t_{n}\right)\right) y & & (n \in N)
\end{aligned}
$$

for some $K \geq 0$ satisfies the inequality

$$
\|U-\varphi(\tau, t, \varepsilon)\| \leq K e^{-\frac{\gamma}{\varepsilon}(\tau-t)} \quad(0 \leq \tau \leq t<\infty)
$$

Using $H_{2}$ and $H_{1}$ we obtain

$$
\|A(t, \varphi(t))\| \leq C
$$


and for $t_{2}>t_{1}$ :

$$
\begin{aligned}
& \left\|A\left(t_{2}, \varphi\left(t_{2}\right)\right)-A\left(t_{1}, \varphi\left(t_{1}\right)\right)\right\| \leq C\left(t_{2}-t_{1}+\left\|\varphi\left(t_{2}\right)-\varphi\left(t_{1}\right)\right\|\right) \\
\leq & C\left(t_{2}-t_{1}+\int_{t_{1}}^{t_{2}}\|F(s, \varphi(s), H(s, \varphi(s), \varepsilon), \varepsilon)\| d s+\sum_{t_{1}<t_{i}<t_{2}}\left\|Q_{i}\left(\varphi\left(t_{i}, \varphi\left(t_{i}\right), \varepsilon\right), \varepsilon\right)\right\|\right) \\
\leq & C\left(t_{2}-t_{1}+C\left(t_{2}-t_{1}\right)+C \sum_{t_{1}<t_{i}<t_{2}} p\right) \leq C(1+3 C)\left(t_{2}-t_{1}\right) .
\end{aligned}
$$

From this estimates and from $\mathrm{H} 3$ follows that Lemma 1 can be applied and consequently exist $K \geq 0$ and $\varepsilon_{1} \geq 0$ such that (5) is satisfied in $\left[0, \varepsilon_{1}\right]$.

If $\varphi(t)$ and $\varphi^{*}(t)$ are two solution of $(4)-\left(4^{\prime \prime}\right)$, then $U_{\varphi}(\tau, t, \varepsilon)-U_{\varphi^{*}}(\tau, t, \varepsilon)$ satisfies the equation

$$
\begin{aligned}
\varepsilon \frac{d}{d s}\left(U_{\varphi}(\tau, s, \varepsilon)-U_{\varphi^{*}}(\tau, s, \varepsilon)\right)= & A(s, \varphi(s))\left(U_{\varphi}(\tau, s, \varepsilon)-U_{\varphi^{*}}(\tau, s, \varepsilon)\right) \\
& \left.\left.+\left(A(s, \varphi(s))-A\left(s, \varphi^{*}(s)\right)\right) U_{\varphi^{*}}(s)\right)\right) U_{\varphi^{*}}(\tau, s, \varepsilon) . \\
\varepsilon \Delta\left(U_{\varphi}\left(\tau, t_{n}, \varepsilon\right)-U_{\varphi^{*}}\left(\tau, t_{n}, \varepsilon\right)\right) / t=t_{n}= & P_{n}\left(\varphi\left(t_{n}\right)\right)\left(U_{\varphi}\left(t_{n}\right)\right)\left(U_{\varphi}\left(\tau, t_{n}, \varepsilon\right)-U_{\varphi^{*}}\left(\tau, t_{n}, \varepsilon\right)\right) \\
& +\left(P_{n}\left(\varphi\left(t_{n}\right)\right)-P_{n}\left(\varphi^{*}\left(t_{n}\right)\right)\right) U_{\varphi^{*}}\left(\tau, t_{n}, \varepsilon\right)
\end{aligned}
$$

Hence

$$
\begin{aligned}
& \left\|U_{\varphi}(\tau, t, \varepsilon)-U_{\varphi^{*}}(\tau, t, \varepsilon)\right\| \\
\leq & \frac{1}{\varepsilon} \int_{t}^{\tau}\left\|U_{\varphi}(\tau, u, \varepsilon)\left(A(u, \varphi(u))-A\left(u, \varphi^{*}(u)\right)\right) U_{\varphi^{*}}(u, t, \varepsilon)\right\| d u \\
& +\frac{1}{\varepsilon} \sum_{t<t_{n}<\tau}\left\|U_{\varphi}\left(\tau, t_{n}, \varepsilon\right)\left(P_{n}\left(\varphi^{*}\left(t_{n}\right)\right)\right) U_{\varphi^{*}}\left(t_{n}, t, \varepsilon\right)\right\| \\
\leq & \frac{K^{2} C}{\varepsilon} e^{-\frac{\gamma}{\varepsilon}(\tau-t)} \int_{t}^{\tau}\left\|\varphi(u)-\varphi^{*}(u)\right\| d u+\frac{K^{2} C F}{\varepsilon} e^{-\frac{\gamma}{\varepsilon}(\tau-t)} \sum_{t, t_{n}<\tau}\left\|\varphi\left(t_{n}\right)-\varphi^{*}\left(t_{n}\right)\right\| \\
\leq & \frac{K^{2} C}{\varepsilon}\left(\left\|x-x^{*}\right\|+\frac{\left\|\left|H-H^{*}\right|\right\|}{1+\Delta}\right) e^{-\frac{\gamma}{\varepsilon}(\gamma-1)}\left(\int_{t}^{\tau}(1+a)^{k(u, \tau)} e^{a(\tau-u)} d u\right. \\
& +\sum_{t<t_{i}<\tau}\left\|\varphi\left(t_{n}\right)-\varphi^{*}\left(t_{n}\right)\right\| \\
\leq & \frac{K^{2}}{\varepsilon}\left(\left\|x-x^{*}\right\|+\frac{\left\|H-H^{*} \mid\right\|}{1+\Delta}\right) e^{\left(a-\frac{\gamma}{\varepsilon}\right)(\tau-t)}\left(\int_{t}^{t_{k}}(1+a)^{n-k+1} e^{-a\left(t_{i}-t\right)} d u\right. \\
& \left.+\sum_{i=k}^{n-1}(1+a)^{n-i} \int_{t_{i}}^{t_{i}+1} e^{-a(u-t)} d u+\int_{t_{n}}^{\tau} e^{-a(u-t)} d u+\sum_{i=k}^{n}(1+a)^{n-i+1} e^{-a\left(t_{i}-t\right)}\right) \\
\leq & \frac{K^{2} C}{\varepsilon}\left(\left\|x-x^{*}\right\|+\frac{\left\|H-H^{*} \mid\right\|}{1+\Delta}\right) e^{\left(a-\frac{\gamma}{\varepsilon}\right)(\tau-t)}\left((1+a)^{n-k+1} \frac{1}{a}+a \sum_{i=k}^{n}(1+a)^{n-1} e^{-a\left(t_{i}-t\right)}\right)
\end{aligned}
$$




$$
\begin{aligned}
\leq & \frac{K^{2} C}{\varepsilon}\left(\left\|x-x^{*}\right\|+\frac{\left\|\left|H-H^{*}\right|\right\|}{1+\Delta}\right) e^{\left(a-\frac{\gamma}{\varepsilon}\right)(\tau-t)}(1+a)^{n-k}(1+a)^{n-k} \\
& \cdot\left(\frac{1+a}{a}+a m \sum_{i=0}^{\infty}(1+a)^{-i} e^{-a l i}\right)
\end{aligned}
$$

Notice that $(n-k) p \leq \tau-t$ and consequently

$$
(1+a)^{n-k}<e^{a(n-k)<e^{\frac{a}{p}}(\tau-t)},
$$

hence

$$
\begin{aligned}
& \left\|U_{\varphi}(\tau, t, \varepsilon)-U_{\varphi^{*}}(\tau, t, \varepsilon)\right\| \\
\leq & \frac{K^{2} C}{\varepsilon}\left(\left\|x-x^{*}\right\|+\frac{\left\|\left|H-H^{*}\right|\right\|}{1+\Delta}\right) e^{\left(\left(1+\frac{1}{p}\right) a-\frac{\gamma}{\varepsilon}\right)(\tau-t)}\left(\frac{1+a}{a}+\frac{a m}{1-(1+a) e^{-a l}}\right)
\end{aligned}
$$

Now we are in position to define a mapping $T$ for every $H \in M(\Delta, D)$.

$$
\begin{aligned}
T(H)(\tau, x, \varepsilon)= & \frac{1}{\varepsilon} \int_{0}^{\tau} U_{\varphi}(\tau, t, \varepsilon) G(t, \varphi(t), H(t, \varphi(t 0, \varepsilon), \varepsilon) d t \\
& \left.+\frac{1}{\varepsilon} \sum_{0<t_{n}<\tau} U_{\varphi}\left(\tau, t_{n}, \varepsilon\right) R_{n}\left(\varphi\left(t_{n}\right), H\left(t_{n}\right), \varphi\left(t_{n}\right), \varepsilon\right), \varepsilon\right)
\end{aligned}
$$

First we show that $T$ maps $M(\Delta, D)$ into $M(\Delta, D)$ :

$$
\begin{aligned}
\|T(H)(\tau, x, \varepsilon)\| & \leq \frac{K C}{\varepsilon}\left(D^{2}+\varepsilon^{2}\right) \int_{0}^{\tau} e^{-\frac{\gamma}{\varepsilon}(\tau-t)} d t+\frac{K C}{\varepsilon}\left(D^{4}+\varepsilon^{4}\right) \sum_{0<t_{n}<\tau} e^{-\frac{\gamma}{\varepsilon}\left(\tau-t_{n}\right)} \\
& \leq \frac{K C}{\gamma}\left(D^{2}+\varepsilon^{2}\right) \int_{0}^{\tau} e^{-\frac{\gamma}{\varepsilon}(\tau-t)} d t+\frac{K C}{\varepsilon}\left(D^{4}+\varepsilon^{4}\right) \frac{m}{1-e^{-\frac{\gamma^{l}}{\varepsilon}}} .
\end{aligned}
$$

Now we suppose that

$$
\left(1+\frac{1}{p}\right) a-\frac{\gamma}{\varepsilon}<0
$$

Then

$$
\begin{aligned}
& \|T(H)(\tau, x, \varepsilon)-T(\bar{H})(\tau, \bar{x}, \varepsilon)\| \\
\leq & \frac{1}{\varepsilon} \int_{0}^{\tau}\left\|U_{\varphi}(\tau, t, \varepsilon)-U_{\bar{\varphi}}(\tau, t, \varepsilon)\right\|\|G(t, \bar{\varphi}(t), \bar{H}(t, \bar{\varphi}(t), \varepsilon), \varepsilon)\| d t \\
& +\frac{1}{\varepsilon} \int_{0}^{\tau}\left\|U_{\varphi}(\tau, t, \varepsilon)-U_{\bar{\varphi}}(\tau, t, \varepsilon)\right\|\|G(t, \bar{\varphi}(t), \bar{H}(t, \bar{\varphi}(t), \varepsilon), \varepsilon)\| d t \\
& +\sum_{0<t_{n}<\tau}\left\|U_{\varphi}\left(\tau, t_{n}, \varepsilon\right)\right\| \| R_{n}\left(\varphi\left(t_{n}\right), H\left(t_{n}\right), H\left(t_{n}, \varphi\left(t_{n}\right), \varepsilon\right), \varepsilon\right. \\
& -R_{n}\left(\bar{\varphi}\left(t_{n}\right), \bar{H}\left(t_{n}, \bar{\varphi}\left(t_{n}\right), \varepsilon\right), \varepsilon\right) \|
\end{aligned}
$$




$$
\begin{aligned}
& +\frac{1}{\varepsilon} \sum_{0<t_{n}<\tau}\left\|U_{\varphi}\left(\tau, t_{n}, \varepsilon\right)-U_{\bar{\varphi}}(\tau, t, \varepsilon)\right\| \| R_{n}\left(\bar{\varphi}\left(t_{n}\right), \bar{H}\left(t_{n}, \bar{\varphi}\left(t_{n}\right), \varepsilon, \varepsilon\right) \|\right. \\
& \leq \frac{K}{\varepsilon}\left(D^{2}+\varepsilon^{2}\right)(1+\Delta)\left(\|x-\bar{x}\|+\frac{\||H-\bar{H}|\|}{1+\Delta}\right) \int_{0}^{\tau}(1+a)^{k(t, \tau)} e^{\left(a-\frac{\gamma}{\varepsilon}\right)(\tau-t)} d t \\
& +\sum_{\frac{K^{2} C}{\varepsilon}}\left(D^{2}+\varepsilon^{2}\right)\left(\frac{1+a}{a}+\frac{a m}{1-(1+a) e^{-a l}}\right)\left(\|x-\bar{x}\|+\frac{\|H-i\|}{1+\Delta}\right) \frac{1}{\gamma-\frac{p+1}{p} a \varepsilon} \\
& +\frac{K}{\varepsilon}\left(D^{2}+\varepsilon^{2}\right)(1+\Delta)\left(\|x-\bar{x}\|+\frac{\||H-\bar{H}|\|}{1+\Delta}\right) \sum_{0<t_{n}<\tau}(1+a)^{k\left(t_{n}, t\right)} e^{\left(a-\frac{\gamma}{\varepsilon}\right)\left(\tau-t_{n}\right)} \\
& +\frac{K^{2} C}{\varepsilon^{2}}\left(D^{4}+\varepsilon^{4}\right)\left(\frac{1+a}{a}+\frac{a m}{1-(1+a) e^{a l}}\right)\left(\|x-\bar{x}\|+\frac{\||H-\bar{H}|\|}{1+\Delta}\right) \sum_{0<t_{n}<\tau} e^{\left(\left(1+\frac{1}{p}\right) a-\frac{\gamma}{\varepsilon}\right)\left(\tau-t_{n}\right)} \\
& \leq \frac{K a}{\gamma-a \varepsilon}\left(D^{2}+\varepsilon^{2}\right)(1+\Delta)\left(\|x-\bar{x}\|+\frac{\||H-\bar{H}|\|}{1+\Delta} \sum_{i=1}^{n}(1+a)^{n+i-1} e^{\left(a-\frac{\gamma}{\varepsilon}\right)\left(\tau-t_{i}\right)}\right. \\
& +\frac{K^{2} C}{\varepsilon}\left(D^{2}+\varepsilon^{2}\right)\left(\frac{1+a}{a}+\frac{a m}{1-(1+a) e^{a l}}\right)\left(\|x-\bar{x}\|+\frac{\||H-\bar{H}|\|}{1+\Delta}\right) \frac{1}{\gamma-\varepsilon \frac{p+1}{p} a} \\
& +\frac{K}{\varepsilon}\left(D^{2}+\varepsilon^{2}\right)(1+\Delta)\left(\| x-\overline{\|}+\frac{\||H-\bar{H}|\|}{1+\Delta}\right) \sum_{0<t_{n}<\tau}^{\left(\left(1+\frac{1}{p}\right) a-\frac{\gamma}{\varepsilon}\right)\left(\tau-t_{n}\right)} \\
& +\frac{K^{2} C}{\varepsilon^{2}}\left(D^{4}+\varepsilon^{4}\right)\left(\frac{1+a}{a}+\frac{a m}{1-(1+a) e^{-a l}}\right)\left(\| x-\overline{\|}+\frac{\||H-\bar{H}|\|}{1+\Delta}\right) \frac{m}{1-e^{\left(\left(1+\frac{1}{p}\right) a-\frac{\gamma}{\varepsilon}\right) l}} \\
& \leq\left(\|x-\bar{x}\|+\frac{\||H-\bar{H}|\|}{1+\Delta}\right)\left\{\frac{K a}{\gamma-a \varepsilon}\left(D^{2}+\varepsilon^{2}\right) \cdot(1+\Delta) \frac{m}{1-e^{\left(\left(1+\frac{1}{p}\right) a-\frac{\gamma}{\varepsilon}\right) l}}\right. \\
& +\frac{K^{2} C}{\varepsilon}\left(D^{2}+\varepsilon^{2}\right)\left(\frac{1+a}{a}+\frac{a m}{1-(1+a) e^{a l}}\right) \frac{1}{\gamma-\varepsilon \frac{p+1}{p} a} \\
& +\frac{K}{\varepsilon}\left(D^{2}+\varepsilon^{2}\right)(1+\Delta) \frac{m}{1-e^{\left(\left(1+\frac{1}{p}\right) a-\frac{\gamma}{\varepsilon}\right) l}} \\
& \left.+\frac{K^{2} C}{\varepsilon^{2}}\left(D^{4}+\varepsilon^{4}\right)\left(\frac{1+a}{a}+\frac{a m}{1-(1+a) e^{-a l}}\right) \frac{m}{1-e^{\left(\left(1+\frac{1}{p}\right) a \frac{\gamma}{\varepsilon}\right) l}}\right\}
\end{aligned}
$$

Now set $D=\varepsilon D_{0}$ and $\Delta=\varepsilon \Delta_{0}$. Hence

$$
\|T(H)(\tau, x, \varepsilon)\| \leq \varepsilon\left(\frac{K C}{\gamma}\left(D_{0}^{2}+1\right)+\varepsilon K C\left(D_{0}^{4}+1\right) \frac{m}{1-e^{-\frac{\gamma^{l}}{\varepsilon}}}\right) .
$$

If $x=\bar{x}$ then

$$
\|T(H)(\tau, x, \varepsilon)-T(\bar{H})(\tau, x, \varepsilon)\| \leq \frac{K a \varepsilon}{\gamma-a \varepsilon}\left(D_{0}^{2}+1\right)(1+\varepsilon \Delta) \frac{m}{1-e^{\left(\left(1+\frac{1}{p}\right) a-\gamma \varepsilon\right) l}}
$$




$$
\begin{aligned}
& +K^{2} C \varepsilon\left(D_{0}^{2}+1\right)\left(\frac{1+a}{a}+\frac{a m}{1-(1+a) e^{-a l}}\right)-\frac{1}{\gamma-\varepsilon \frac{p+1}{p} a} \\
& +K \varepsilon\left(D_{0}^{2}+1\right)\left(1+\varepsilon \Delta_{0}\right) \frac{m}{1-e^{\left((1+1-\bar{p}) a-\frac{\gamma}{\varepsilon}\right) l}} \\
& \left.+K^{2} C \varepsilon\left(D_{0}^{4}\right)\left(\frac{1+a}{a}+\frac{a m}{1-(1+a) e^{-a l}}\right) \frac{m}{1-e^{\left(\left(1+\frac{1}{p}\right) a-\frac{\gamma}{\varepsilon}\right) l}}\right\} \frac{\||H-\bar{H}|\|}{1+\varepsilon \Delta_{0}} .
\end{aligned}
$$

If $H=\bar{H}$ then

$$
\begin{aligned}
& \|T(H)(\tau, x, \varepsilon)-T(H)(\tau, \bar{x}, \varepsilon)\| \leq\left\{\frac{K a \varepsilon^{2}}{\gamma-a \varepsilon}\left(D_{0}^{2}+1\right)\left(1+\varepsilon \Delta_{0}\right) \frac{m}{1-e^{\left(\left(1+\frac{1}{p}\right) a-\gamma \varepsilon\right) l}}\right. \\
& +K^{2} C \varepsilon\left(D_{0}^{2}+1\right)\left(\frac{1+a}{a}+\frac{a m}{1-(1+a) e^{-a l}}\right)-\frac{1}{\gamma-\varepsilon^{\frac{p+1}{p} a}} \\
& +K \varepsilon\left(D_{0}^{2}+1\right)\left(1+\varepsilon \Delta_{0}\right) \frac{m}{1-e^{\left((1+1 \bar{p}) a-\frac{\gamma}{\varepsilon}\right) l}} \\
& \left.+K^{2} C \varepsilon^{2}\left(D_{0}^{4}\right)\left(\frac{1+a}{a}+\frac{a m}{1-(1+a) e^{-a l}}\right) \frac{m}{1-e^{\left(\left(1+\frac{1}{p}\right) a-\frac{\gamma}{\varepsilon}\right) l}}\right\}\|x-\bar{x}\| .
\end{aligned}
$$

Set $a=\frac{\gamma p}{2 \varepsilon(p+1)}$. There exists $\varepsilon_{3}$ such that for every $\varepsilon \in\left(0, \varepsilon_{3}\right]$ the inequation

$$
C\left(1+\varepsilon \Delta_{0}\right) \leq \frac{\gamma p}{2 \varepsilon(p+1)}
$$

holds. Next we find $D_{0}$ such that inequation

$$
\varepsilon^{2}\left(\frac{K C}{\gamma}\left(D_{0}^{2}+1\right)+\varepsilon K C\left(D_{0}^{4}+1\right) \frac{m}{1-e^{-\frac{\gamma^{l}}{\varepsilon}}}\right) \leq \varepsilon D_{0}
$$

holds true for every $\varepsilon \in\left(0, \varepsilon_{3}\right]$. Then from (7) follows that $\|T(H)(\tau, x, \varepsilon)\| \leq D$ in $\Omega$. Finally we find $\Delta_{0}$ such that inequation

$$
\begin{aligned}
& \varepsilon \frac{K p}{p+2}\left(D_{0}^{2}+1\right)\left(1+\varepsilon \Delta_{0}\right) \frac{m}{1-e^{-\frac{\gamma}{2 \varepsilon} l}}+K^{2} C \varepsilon\left(D_{0}^{2}+1\right)\left(\frac{2 \varepsilon^{2}(p+1)+\gamma p \varepsilon}{\gamma(p+1)}\right. \\
& +\frac{\gamma m p}{2(p+1)\left(1-\frac{\gamma p+2 \varepsilon(p+1)}{2 \varepsilon(p+1)} e^{-\frac{\gamma p}{2 \varepsilon(p+1)} l}\right)} \frac{2}{\gamma}+K \varepsilon\left(D_{0}^{2}+1\right)\left(1+\varepsilon \Delta_{0}\right) \frac{m}{1-e^{-2 \frac{\gamma}{\varepsilon} 1}} \\
& +K^{2} C \varepsilon\left(D_{0}^{4}+1\right)\left(\frac{2 \varepsilon^{2}(p+1)+\gamma p \varepsilon}{\gamma(p+1)}\right. \\
& \left.\left.+\frac{\gamma m p}{2(p+1)\left(1-\frac{\gamma p+2 \varepsilon(p+1)}{2 \varepsilon(p+1)} e^{-\frac{\gamma p}{2 \varepsilon(p+1)} l}\right)}\right)\right\} \frac{m}{1-e^{-2 \frac{\gamma}{\varepsilon} l} \leq \varepsilon \Delta_{0}}
\end{aligned}
$$

holds true for every $\varepsilon \in\left(0, \varepsilon_{3}\right]$. Then from (9) follows that

$$
\|T(H)(\tau, x, \varepsilon)-T(H)(\tau, \bar{x}, \varepsilon)\| \leq \Delta\|x-\bar{x}\|
$$


in $\Omega$ and consequently $T(H) \in M(\Delta, D)$. Now let show that $T(H)$ is a contraction on $M(\Delta, D)$ providing a unique fixed point $H^{*}$. In fact, from (8) we receive the estimate

$$
\begin{aligned}
& \|T(H)(\tau, x, \varepsilon)-T(\bar{H})(\tau, x, \varepsilon)\| \leq\left\{\varepsilon \frac{K p}{p+2}\left(D_{0}^{2}+1\right)\left(1+\varepsilon \Delta_{0}\right) \frac{m}{1-e^{-\frac{\gamma}{2 \varepsilon} 1}}\right. \\
& +K^{2} C \varepsilon\left(D_{0}^{2}+1\right)\left(\frac{2 \varepsilon^{2}(p+1)+\gamma p \varepsilon}{\gamma(p+1)}+\frac{\gamma m p}{2(p+1)\left(1-\frac{\gamma p+2 \varepsilon(p+1)}{2 \varepsilon(p+1)} e^{-\frac{\gamma p}{2 \varepsilon(p+1)} l}\right)}\right) \frac{2}{\gamma} \\
& +K \varepsilon\left(D_{0}^{2}+1\right)\left(1+\varepsilon \Delta_{0}\right) \frac{m}{1-e^{-2 \frac{\gamma}{\varepsilon} l}}+K^{2} C \varepsilon\left(D_{0}^{4}+1\right)\left(\frac{2 \varepsilon^{2}(p+1)+\gamma p \varepsilon}{\gamma(p+1)}\right. \\
& \left.\left.+\frac{\gamma m p}{2(p+1)\left(1-\frac{\gamma p+2 \varepsilon(p+1)}{2 \varepsilon(p+1)} e^{-\frac{\gamma p}{2 \varepsilon(p+1)} l}\right)}\right) \frac{m}{1-e^{-2 \frac{\gamma}{\varepsilon} l}}\right\} \frac{\|H-\bar{H} \mid\|}{1+\varepsilon \Delta_{0}} \\
& \leq \frac{\varepsilon \Delta_{0}}{1+\varepsilon \Delta_{0}}\||H-\bar{H}|\| \leq \frac{\varepsilon_{3} \Delta_{0}}{1+\varepsilon_{3} \Delta_{0}}\||H-\bar{H}|\| .
\end{aligned}
$$

This completes the proof.

\section{References}

[1] D. D. Bainov, S. I. Kostadinov, Abstract Impulsive Differential Equations, SCT Publishing, 1994.

[2] D. D. Bainov, S. I. Kostadinov, Nguyen Van Minh, P. P. Zabreiko, "Continuous dependence on a parameter of the solutions of impulisve differential equations in a Banach space," International Journal of Theoretical Physics, 32(7)(1993).

[3] D. D. Bainov, S. I. Kostadinov, Nguyen Van Minh, Dichotomies and Integral Manifolds of Impulsive Differential Equations, SCT Publishing, 1994.

[4] D. D. Bainov, S. I. Kostadinov, P. P. Zabreiko, " $L_{p}$-equivalence of linear and nonlinear impulsive differential equation in a Banach space," Journal of Mathematical Analysis and Applications, 159(2)(1991), 389-405.

[5] A. M. Samoilenko, N. A. Perestyuk, Differential Equations with Impulsive Effect, Vista Skola, Kiev (1987), 285pp. (in Russian).

[6] V. V. Strygin, V. A. Sobolev, Separation of the Motion by the Method of Integral Manifolds, 1988 (in Russian).

${ }^{1,3}$ University of Plovdiv, Bulgaria.

${ }^{2}$ Weierstrass Institute for Analysis and Stochastics, Berlin, Germany. 